\title{
UMA INVESTIGAÇÃO BASEADA EM CORPUS SOBRE PADRÕES DE ESTILO DE UM TRADUTOR LITERÁRIO RELACIONADOS AO CONECTIVO THAT/ZERO E A FORMAS FIXAS E SEMI-FIXAS, OBSERVADOS EM DISCOVERING THE WORLD
}

\section{Diva Cardoso de Camargo*}

RESUMO: Este estudo tem por tema o estilo de um tradutor literário, relacionado ao uso individual de padrões recorrentes de comportamento lingüístico. Considerando que a definição de estilo não chegou a um consenso mesmo para as disciplinas da crítica literária e da estilística, adotou-se a noção proposta por Baker (2000: 246-7), que concebe estilo como uma espécie de impressão digital, que fica expressa no texto traduzido por uma variedade de características lingüísticas. A fim de investigar o estilo do tradutor literário Giovanni Pontiero, compilei para o corpus principal a tradução Discovering the World feita por esse profissional, e para o corpus de referência o respectivo original A Descoberta do Mundo, de Clarice Lispector. Em contraste com o modo tradicional de examinar se o estilo do autor foi adequadamente transposto na tradução, este estudo teve por objetivo identificar, no corpus de tradução, o uso de um estilo distintivo da parte de Pontiero, evidenciado por padrões recorrentes e preferenciais, envolvendo estruturas do verbo de elocução SAY. O trabalho foi desenvolvido semi-automaticamente, por meio de uma combinação de análise computadorizada, utilizando os programas WordSmith Tools e Multiconcord, e análise manual. A observação de padrões de estilo individuais, recorrentes e preferenciais revela escolhas estilisticas, feitas consciente ou inconscientemente por Pontiero, refe-

* Departamento de Letras Modernas da UNESP/SJRP. 
rentes ao uso de padrões com as formas de SAY envolvendo tanto o conectivo opcional that/zero como expressões fixas e semi-fixas, todos esses padrões com freqüências mais altas do que em relação ao respectivo texto original.

UNITERMOS: tradução literária; estudo de tradução baseado em corpus; estilo do tradutor; Giovanni Pontier; literatura brasileira traduzida; Clarice Lispector.

ABSTRACT: The topic of this study is the literary translator's style, related to the individual use of recurrent patterns of linguistic behaviour. Considering the lack of a consensual definition for style even in the fields of literary criticism and stylistics, this article drew upon Baker's understanding of style (2000: 246-7) as a 'fingerprint' identifiable in the translated text through a variety of linguistic elements. In order to investigate the style of the literary translator Giovanni Pontiero, I adopted his English translation Discovering the World as the main corpus and Clarice Lispector's original Brazilian fiction A Descoberta do Mundo as the reference corpus. Instead of examining whether the author's style was adequately transferred to the target text, this article aims to identify Pontiero's distinctive style as evidenced in the translated text corpus by the use of recurring patterns involving structures of the reporting verb SAY. The task was carried out semi-automatically in a combination of manual and computerized analysis performed with the WordSmith Tools and Multiconcord software packages. The observation of individual, recurrent and preferred stylistic patterns revealed Pontiero's stylistic choices, either conscious or unconscious, of the patterns of SAY involving both the optional that/zero and patterns of fixed and semi-fixed expressions, all of which occurred more frequently than in the corresponding source text.

KEYWORDS: literary translation, corpus-based translation research, translator's style, Giovanni Pontiero, translated Brazilian literature, Clarice Lispector. 


\section{Introdução}

Ainda hoje a conceituação de estilo não obteve um consenso geral por parte das disciplinas da Crítica Literária e da Estilística. Também o mesmo ocorre quanto ao emprego de concepções de estilo para a tradução, porquanto as várias tentativas têm abordado as escolhas 'boas' ou 'más' feitas por determinados tradutores ou, mais freqüentemente, têm prescrito regras para a seleção de estratégias tradutórias específicas a partir de tipos de texto ou registro. Esse fato reflete que, tanto nos estudos literários como nos estudos lingüísticos, a noção de estilo está tradicionalmente associada quer a um dado escritor ou orador (ex.: o estilo de Steinbeck, Guimarães Rosa, Graciliano Ramos; Winston Churchill, Joaquim Nabuco, Rui Barbosa), quer a características estilísticas específicas de textos produzidos num dado período literário (ex.: Barroco, Romantismo, Modernismo), quer a características lingüísticas associadas a textos produzidos por grupos específicos de usuários da língua e num contexto institucional específico (ex.: o estilo de editoriais, patentes, sermões). Em decorrência, os estudos da tradução herdaram dos estudos literários a valorização do 'original' e a preocupação com o estilo do autor, sua individualidade e criatividade, mas somente para descrever como as características estilísticas do escritor poderiam elucidar o processo de tradução da sua obra. Subjacente a tais associações com a escritura 'original', caberia ao tradutor a [impossivel] incumbência de não ter estilo próprio, e simplesmente reproduzir, da maneira a mais impessoal, o estilo do autor. Da lingüística, foi passado para os estudos da tradução o interesse pela observação do estilo de grupos sociais de usuários da lingua, a fim de analisar como os registros lingüísticos (ex.: jargão médico, discurso jurídico, linguagem literária) ou como as características de tipos de texto diversos (ex.: textos jornalísticos, corporativos, literários) podem trazer informações para o ato tradutório. Tais estudos visam, em sua maioria, a oferecer um ponto de partida para identificar características distintivas do texto original (TO), a fim de reproduzi-las na tradução ou observar desvios da norma por parte do tradutor.

Até recentemente, pouco se havia investigado sobre o estilo de determinado tradutor, ou grupo de tradutores, ou mesmo 
um corpus de material traduzido que pertença a um dado período literário. É só nas últimas décadas que a presença do tradutor no texto ou, mais especificamente, de traços individuais que essa presença deixa no texto, começou a receber certa atenção da literatura sobre tradução. Contudo, o enfoque tem-se ainda restringido: a) para a avaliação da qualidade das traduções; b) para a descrição de tendências gerais do texto traduzido (TT) em relação ao TO, mas ignorando as idiossincrasias do tradutor; c) para a descrição da intervenção do tradutor apenas no tocante a acréscimos de material paratextual ou glossários.

Dentre os modelos mais conhecidos, tem-se o de House $(1977,1981,1997)$ que descreve o texto fonte $(\mathrm{TF})$ quanto a peculiaridades lingüísticas (dimensão do usuário da língua) e peculiaridades situacionais (dimensão dos usos da língua), comparando TF e o texto meta quanto a aproximações relativas entre ambos. Todavia, consoante a afirmação de Baker (2000: 242) esse modelo não fornece um tratamento sistemático da noção de estilo, porquanto o que permite descrever não seria tanto o estilo do TF ou do autor, e também não seria o estilo da tradução ou do tradutor, mas os pontos onde os dois textos divergem ao longo apenas das duas dimensões mencionadas.

Nos últimos anos, alguns teóricos da tradução têm enfatizado a presença do tradutor; no entanto, não apresentam nenhuma demonstração dos traços efetivamente deixados em seus TTs. Venuti $(1992,1995,1998)$ recrimina a transparência como efeito ilusionístico da presença do autor que seria [supostamente] alcançada pelas estratégias da tradução domesticadora e advoga a visibilidade do tradutor por meio de estratégias de resistência da tradução estrangeirizadora, mas sem explicitar quais seriam as marcas de uma 'fidelidade abusiva'. Analogamente, Hermans (1996) claramente reconhece a voz do tradutor; porém, focaliza principalmente a 'voz do outro' no que tange ao emprego auto-referencial de primeira pessoa nas notas do tradutor.

No que concerne à tradução, a noção de estilo poderia incluir a escolha pelo tradutor de material a ser traduzido, a utilização consistente de estratégias tradutórias da parte de cada tradutor (incluindo o uso de prefácios, glossários, notas de ro- 
dapé, etc.) e, sobretudo, o modo de expressão que é típico de um dado tradutor (mais do que simplesmente instâncias de intervenção aberta de material extratextual).

Dado que o conceito de estilo tem-se mostrado de dificil definição, este estudo do estilo do tradutor literário optou por fundamentar-se na noção fornecida por Baker, que entende

estilo como uma espécie de impressão digital que fica expressa [no TT] por uma variedade de características lingüisticas (...) as quais estão provavelmente mais no domínio do que algumas vezes é chamado de 'estilística forênsica' que no da estilística literária (Leech \& Short 1981: 14). Tradicionalmente, a estilística literária focaliza o que se assume serem escolhas lingüisticas conscientes da parte do autor, porque os estilistas literários estão principalmente interessados na relação entre as características lingüísticas e a função artística, em como um dado autor obtém certos efeitos artísticos. Por outro lado, a estilistica forênsica tende a focalizar hábitos lingüísticos razoavelmente sutis e moderados que estão bem acima do controle consciente do autor e que nós, como receptores, registramos, na maioria das vezes, de forma subliminar. Todavia, como ambos os ramos da estilística, estou interessada em padrões de escolha (quer essas escolhas sejam conscientes ou subconscientes) mais do que em escolhas individuais isoladas. ${ }^{1}$ (2000: 246-7).

Dessa forma, este trabalho não está voltado para o sentido tradicional de examinar se o estilo do autor foi adequadamente transposto 'na' tradução, mas, sim, direciona o foco para um estilo 'de' tradução. Apesar das dificuldades que a investigação de um estilo 'de' tradução possa acarretar, no que tange à disciplina a importância dessa mudança de enfoque aponta para a necessidade de estudos que proponham uma nova abordagem. E que esta possibilite a identificação da presença do tradutor no TT, referente ao uso de padrões estilísticos próprios,

1 Esta e todas as outras traduções do inglês são minhas. 
independentemente do estilo do autor do TO e do par lingüístico envolvido.

Com o propósito de observar o perfil estilístico do tradutor literário em questão, isso foi definido, no âmbito deste estudo, como o perfil de seus hábitos lingüísticos individuais, preferenciais, recorrentes e distintivos, referentes a estruturas do verbo de elocução SAY (em todas as suas formas: say, says, said, saying) envolvendo tanto o conectivo opcional that/zero como expressões fixas e semi-fixas que são tipicamente empregadas para explicitação ou explicação (ex.: that is to say, needless to say, was nothing more to be said, as I said before, it goes without saying, as I was saying, etc.).

\section{Perspectiva teórica}

A idéia de se elaborar um corpus de tradução partiu do questionamento sobre "o porquê de os TTs serem considerados nada mais que versões distorcidas e de segunda-mão dos textos 'verdadeiros' “, segundo relata Baker (1993: 233); também acrescenta que, até então, os TTs só eram considerados nos casos em que se buscava mostrar exemplos de translationese, ou seja, da influência de outra língua na tradução (1996: 178). Para a autora, a prova mais evidente provém da própria disciplina Lingüística de Corpus, que exclui os TTs de corpora monolíngües por não serem considerados representativos da língua em estudo. $\mathrm{Na}$ opinião de Baker (1995), o fato de a visão tradicional considerar o ato tradutório como diferente de outros atos comunicativos mostra que, ao invés da pouca atenção que lhe é dada, é justamente a natureza dessas diferenças que deve ser registrada e explorada. Partindo das conquistas de Even-Zohar (1978, 2000: 192-197) e, sobretudo, de Toury (1978, 2000: 198-211), relativas ao enfoque no sistema da literatura traduzida e nas normas que governam esses sistemas, vale-se também Baker (1993) de uma nova 'virada' dentro dos estudos lingüísticos para fundamentar a constituição da disciplina de estudos da tradução por meio da natureza do TT.

Devido à mudança de uma perspectiva conceitual e semântica da linguagem (com estudos baseados na introspecção) 
para uma perspectiva situacional e de uso da linguagem (com estudos voltados para o contexto), essa 'virada' leva a uma mudança nos métodos de investigação nos estudos lingüísticos, passando a solicitar o acesso a dados reais e a uma grande quantidade de dados para observações sobre o uso da linguagem. Desse modo, os estudos da tradução passam a receber subsídios de outra vertente fornecida pela Lingüística de Corpus (LC), especialmente pelas contribuições de Sinclair. Por meio de uma coleção de corpora computadorizados para estudos lexicológicos, bem como do desenvolvimento de uma metodologia de pesquisa que possibilita investigações acima das limitações humanas e minimiza a dependência da intuição, Sinclair (1991) traçou os caminhos da maioria das pesquisas em LC feitas até hoje.

Dado o avanço que a LC trouxe para a análise de TTs, na opinião de Berber Sardinha (no prelo), já haveria maior unanimidade entre os pesquisadores da tradução e os lingüistas de corpus a respeito da exploração de corpora eletrônicos para a disciplina emergente de Estudos da Tradução. De acordo com o autor (ibidem), os resultados alcançados com esse tipo de observação têm demonstrado que "a organização da linguagem é muito mais complexa do que o suposto, ficando claro que a utilização de corpora, de certa forma, deixou de ser uma opção para investigações na área".

Outrossim, a utilização de corpora em formato eletrônico para os Estudos da Tradução é valorizada por Tymoczko (1998), ao afirmar que essa nova abordagem é importante para a sustentação e o desenvolvimento da disciplina neste século. A autora ressalta a importância de se visualizar o maior número possível de tipos de corpora para serem empregados em pesquisas em tradução, bem como enfatiza a necessidade de se estabelecer questões que possam ser investigadas num corpus, além de métodos que possam ser utilizados para se obter tanto resultados teóricos quanto práticos. Destaca, ademais, que um corpus computadorizado é uma forma eficiente para armazenar, acessar e pesquisar uma vasta quantidade de informações, muito maior do que seria possivel a um ser humano examinar manualmente, sem o auxílio de ferramentas eletrônicas. Complementa, ainda, que os corpora computadorizados aumentam a qualidade e a 
quantidade do conteúdo e dos métodos da disciplina, adequando-a aos novos padrões dessa era da informação.

Em consonância com os teóricos que se valeram da LC para os estudos da tradução, Baker (1993) afirma que os grandes corpora oferecem

\begin{abstract}
uma oportunidade única para observar o seu objeto de estudo e explorar o que o diferencia de outros objetos de estudo, como a língua em geral ou outros tipos de interação cultural. Também permitirão, em uma escala maior do que era possivel anteriormente, explorar os principios que governam o comportamento tradutório e as amarras em que opera (p. 235).
\end{abstract}

Partindo do reconhecimento da tradução enquanto espaço diferencial que deve ser privilegiado na cultura de chegada, Baker considera a tradução como objeto de pesquisa per se, e elege, como quadro metodológico para pesquisa, a abordagem da LC. Nesse sentido, Baker (ibidem, p. 243) destaca que "a tarefa mais importante que aguarda a aplicação das técnicas de corpus nos estudos da tradução (...) é a elucidação da natureza do texto traduzido como um evento comunicativo mediado". Para que isso possa ocorrer, prossegue Baker, torna-se necessário o uso de corpora em formato eletrônico em um grande número de textos. Então, tendo-se inspirado no lingüista pioneiro Sinclair, a autora afirma que a verdadeira 'virada' na história da disciplina acontecerá “(...) como conseqüência direta do acesso a grandes corpora de textos originais e traduzidos, e do desenvolvimento de métodos específicos e de ferramentas que permitam investigar esses corpora de modo adequado às necessidades dos pesquisadores da área" (ibidem, p. 235).

Algumas generalizações foram propostas em virtude de a utilização de corpora eletrônicos paralelos ou comparáveis ${ }^{2}$ possi-

2 Um corpus paralelo é composto de $\mathrm{TO}(\mathrm{s})$ em uma determinada língua (lingua de origem) e sua(s) respectiva(s) tradução(ões) em outra língua (língua de tradução). Um corpus comparável, por sua vez, consiste em dois conjuntos de textos em uma mesma lingua: um composto de TOs e

TRadTerm, 11, 2005, p. 107-131 
bilitar maior amplitude e funcionalidade para estudos da natureza da tradução. Dentre as generalizações possiveis, as investigações lideradas por Baker (1996: 180-184) na UMIST e na Universidade de Manchester desde junho de 2003 têm detectado certas características ou traços recorrentes que se apresentam tipicamente nos TTs. São alguns deles:

1. Explicitação: tendência geral em explicar e expandir dados do TO. Manifestações dessa tendência podem ser observadas habitualmente nos TTs em relação aos TOs, como a sua maior extensão, o emprego exagerado de vocabulário e de conjunções e locuções conjuntivas coordenativas explicativas.

2. Simplificacão: tendência em tornar mais simples e de mais fácil compreensão a linguagem empregada na tradução. Evidências podem ser encontradas nos TTs em relação aos TOs, como repetição de palavras e mudança na pontuação, para trazer maior clareza ao enunciado. Uma medida possivel de traços de simplificação é fornecida pela razão forma/ocorrência (type/token ratio) e densidade lexical.

3. Normalização ou conservacionismo: tendência para exagerar características da língua meta e para adequar-se aos seus padrões típicos. Evidências podem ser notadas no uso de clichês e estruturas gramaticais convencionais nos TTs.

4. Estabilização: tendência para a tradução localizar-se, independentemente das línguas fonte e meta, no centro de um contínuo, evitando-se os extremos. Manifestações podem ser encontradas, por exemplo, na tendência de os tradutores empregarem a norma culta nas marcas da linguagem oral utilizadas pelo autor para caracterizar determinados personagens.

O enfoque comparativo em corpora eletrônicos paralelos ou comparáveis têm contribuído ativamente para a teoria da tradução, ao procurar elucidar a natureza dos TTs e o processo tradutório, objetos esses essenciais para delimitar os objetivos e

outro de TTs para a língua em questão (por exemplo: o inglês), a partir de uma única língua fonte (por exemplo: o português) ou de diversas línguas fonte (por exemplo: o português, francês, etc.). 
a área de atuação da disciplina. Dessa maneira, ao invés de exames de TTs voltados apenas para o levantamento de características distintivas do TO, a fim de reproduzi-las na tradução, ou do levantamento de desvios da norma por parte do tradutor, novos horizontes têm sido abertos aos estudos de tradução pela LC, permitindo que se proponham diferentes investigações sobre o estilo de determinado tradutor, ou grupo de tradutores, ou mesmo corpus de material traduzido que pertença a um dado período literário. Como decorrência, a presença do tradutor no texto ou, mais especificamente, de traços individuais dessa presença poderão vir a fazer parte da literatura sobre tradução. Em virtude de uma tendência dos Estudos de Tradução baseados em corpora valer-se de exemplos autênticos, seria uma das possiveis abordagens que poderia, talvez, vir a diminuir um pouco a suspeita de uma boa parte dos tradutores profissionais em relação à Teoria da Tradução. Nesse sentido, a pesquisa de corpora de TTs tem trazido importantes contribuições para a prática tradutória. Em lugar de um direcionamento mais prescritivo recomendando a reprodução 'fiel' do TO e criticando 'desvios' do tradutor, um destaque é a preocupação muito mais descritiva, com a mudança do foco para o que o tradutor realmente faz na língua de chegada, observando o uso de padrões individuais referentes aes referentes esvios"iticando "a reprodução "traços próprios, distintivos, preferenciais e recorrentes deixados por ele nas suas traduções.

No entanto, existem certas limitações para se iniciar uma pesquisa em corpus paralelo, dentre elas a necessidade de embasamento teórico-metodológico e treinamento específico, preparação dos textos em formato adequado de análise, além de subsídios financeiros para a aquisição de equipamentos avançados de computação, de software de análise (ex.: WordSmith Tools, Multiconcord, etc.) e de CD-ROM.

Apesar dessas dificuldades, os corpora em formato eletrônico analisados com ferramentas computacionais podem oferecer uma série de vantagens para pesquisas em tradução, como as elencadas por Tagnin (2001): manipulação de grande quantidade de dados; acesso a dados autênticos, observáveis e verificáveis; repetição de uma experiência e confirmação de intuições 
e conclusões; estudos de freqüência sobre dados encontrados e não-encontrados; obtenção de dados quantitativos/estatísticos; e generalizações.

Dessa forma, os estudos de TTs por meio de corpora computadorizados são de relevância para a disciplina emergente de Estudos da Tradução, não só por partirem do novo conceito teórico de pesquisa descritiva baseada na língua em uso, mas também por aceitarem os principios da análise de corpus como metodologia.

\section{Resultados e discussão}

Com o propósito de observar, dentro do conceito de estilo adotado no âmbito desta investigação, alguns traços de hábitos lingüísticos próprios e recorrentes, característicos do profissional em estudo, examinei, por meio do verbo dicendi SAY, padrões de escolha encontrados na tradução compilada no corpus principal.

No tocante aos verbos dicendi ou de elocução, suas estruturas são importantes para análise, por poder-se esperar um alto nivel de variação, dado que cada narrador transmite, de modos diversos, o pensamento expresso pelos diferentes personagens, servindo-se dos discursos direto, indireto ou indireto livre. No entanto, dentro do escopo deste trabalho, não seria possivel explorar mais de um verbo dicendi no discurso indireto, devido à sua alta freqüência e à necessidade de observação meticulosa de um grande número de concordâncias. Dado que o verbo de elocução SAY apresenta o maior emprego na língua inglesa, optei, assim, por analisar suas estruturas com construções de inclusão e omissão do conectivo optional that bem como com expressões fixas e semi-fixas.

\section{Alguns padrões de escolha do tradutor em estruturas do verbo SAY + THAT/ZERO}

Em virtude de o uso opcional da conjunção subordinativa integrante that (também denominada de conectivo opcional that, 
conectivo that/zero, ou conectivo that/ $\varnothing$ ) em discursos indiretos em inglês não ocorrer no português, um exame da utilização desse conectivo poderia oferecer possibilidades de identificação de padrões distintivos de escolha do tradutor em questão. Dessa maneira, variáveis relacionadas com a influência da língua de partida, da obra original e do estilo do autor poderiam apresentar menor probabilidade de interferência na obra traduzida e no comportamento lingüístico desse profissional.

Com referência a evidências do conectivo that/zero em estudos anteriores, destaco, dentre eles, os realizados junto ao CTIS por Burnett (1999) e por Baker \& Olohan (2000), os quais se valem de comparações entre o Translational English Corpus (TEC) e o British National Corpus (BNC).

Os resultados obtidos por essas duas investigações revelam que os textos traduzidos para o inglês, provenientes de diferentes linguas de partida, apresentam uma tendência bastante marcada no uso do conectivo that com o verbo SAY, além de outros verbos de elocução. Inversamente, os dados mostram freqüências mais altas e constantes do conectivo zero em textos originalmente escritos em inglês. A título de ilustração, reproduzo, na Tabela 1, abaixo, os dados referentes ao verbo SAY, relatados por Baker e Olohan (ibidem, p. 153):

\begin{tabular}{|l|l|l|l|l|l|l|l|l|}
\hline & $\begin{array}{l}\text { say } \\
(\mathrm{BNC})\end{array}$ & $\begin{array}{l}\text { say } \\
(\mathrm{TEC})\end{array}$ & $\begin{array}{l}\text { says } \\
(\mathrm{BNC})\end{array}$ & $\begin{array}{l}\text { says } \\
(\mathrm{TEC})\end{array}$ & $\begin{array}{l}\text { said } \\
(\mathrm{BNC})\end{array}$ & $\begin{array}{l}\text { said } \\
(\mathrm{TEC})\end{array}$ & $\begin{array}{l}\text { saying } \\
(\mathrm{BNC})\end{array}$ & $\begin{array}{l}\text { saying } \\
(\text { TEC) }\end{array}$ \\
\hline That & 323 & 316 & 64 & 116 & 183 & 267 & 142 & 76 \\
\hline$\%$ & $\mathbf{2 6 , 5}$ & $\mathbf{5 5 , 5}$ & $\mathbf{1 2 , 8}$ & $\mathbf{4 0 , 4}$ & $\mathbf{1 9 , 2}$ & $\mathbf{4 6 , 5}$ & $\mathbf{4 3 , 0}$ & $\mathbf{6 7 , 2}$ \\
\hline Zero & 895 & 253 & 435 & 171 & 771 & 307 & 188 & 37 \\
\hline$\%$ & $\mathbf{7 3 , 5}$ & $\mathbf{4 4 , 5}$ & $\mathbf{8 7 , 2}$ & $\mathbf{5 9 , 6}$ & $\mathbf{8 0 , 8}$ & $\mathbf{5 3 , 5}$ & $\mathbf{5 7 , 0}$ & $\mathbf{3 2 , 7}$ \\
\hline
\end{tabular}

Tabela 1: Distribuição do conectivo that/zero em TOs em inglês compilados no BNC e em TTs para o inglês compilados no TEC

Dentro dessa perspectiva, utilizei a ferramenta Concord, disponibilizada pelo programa WordSmith Tools, a fim de produzir, a partir da tradução, concordâncias ou listagens de ocorrências da palavra de busca SAY em todas as suas formas: say, says, said, saying. Foram consideradas para exame detalhado somente as linhas que apresentaram associações com as duas construções selecionadas, referentes a: SAY + that e SAY + zero. No Apêndice 1, encontram-se listadas as linhas de concordâncias selecionadas. 
De modo geral, as duas construções introduzem, no discurso indireto em inglês, orações subordinadas substantivas objetivas diretas como, por exemplo, nas concordâncias abaixo, extraídas de Discovering the World:

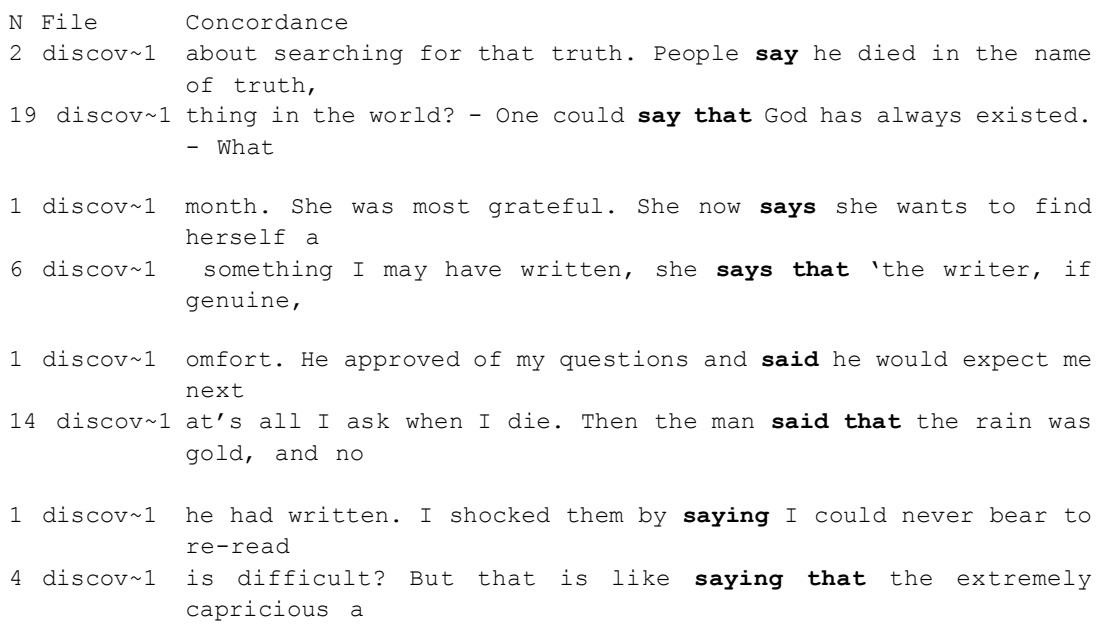

De um total de 444 linhas de concordâncias geradas no TT, 69 foram identificadas com construções that/zero. Em decorrência, foi descartado um número considerável de linhas de concordâncias com SAY, por não conter essas construções, ou seja, por serem irrelevantes para a análise neste momento. Neste caso, foram, em geral, descartados os seguintes tipos de construções:

objetos diretos não-oracionais

dw Every word in the book was essentially meant to say something. And I still think of my

sintagmas preposicionados

dw e could say of my novel what is often said about other people's lives:

'But wh

orações subordinadas adverbiais

dw The little room, as I told you, was furnished.It was difficult to say if the arrangement

orações interrogativas indiretas

dw adapting to God's freedom. We cannot say how we might have turned out if we had been 
orações reduzidas

dw is moving in that direction - or as the moralists would say turning to higher things - I

orações interferentes

dw But now and then, like 'a wild colt', as Eva might say, she would go off on some expressões fixas

dw would I still have become a novelist, that is to say, a novelist writing in English? In

formas de discurso direto

dw was writing! I interrupted her to say: -But you've written the story in my very own

dw ark. - I am going to tell a story, he said, and you will write a composition. dw ions throughout Brazil. - For a time, said Gisela, I myself worked in a satell

Após a contagem manual das concordâncias, extraí a Tabela 2 , que mostra, em valores absolutos e percentuais, o uso do conectivo that e zero para cada forma de SAY:

\begin{tabular}{|l|l|l|l|l|}
\hline & \multicolumn{4}{|l|}{$\begin{array}{l}\text { CORPUS PRINCIPAL } \\
\text { (TRADUÇÃO POR PONTIERO DE Discovering the World, DE LISPECTOR) } \\
\text { Total de palavras: } 193.891\end{array}$} \\
\hline & $+\varnothing$ & $\%$ & + that & $\%$ \\
\hline Say & 18 & $\mathbf{4 3 , 9}$ & 23 & $\mathbf{5 6 , 1}$ \\
\hline Says & 5 & $\mathbf{8 3 , 3}$ & 1 & $\mathbf{1 6 , 7}$ \\
\hline Said & 11 & $\mathbf{6 8 , 8}$ & 5 & $\mathbf{3 1 , 2}$ \\
\hline Saying & 4 & $\mathbf{8 0 , 0}$ & 1 & $\mathbf{2 0 , 0}$ \\
\hline
\end{tabular}

Tabela 2: Concordâncias com SAY + that/zero no TT de Pontiero/Lispector

Tomando como base a tradução de Discovering the World, os resultados da Tabela 2 destacam maior freqüência no uso do conectivo optional that apenas com referência à forma say $(56,1 \%)$. Essa porcentagem mais alta parece confirmar a expectativa de maior uso do conectivo that na linguagem empregada nos textos traduzidos, segundo as pesquisa de Burnett (1999) e Baker \& Olohan (2000), acima. Esse resultado também evidenciaria uma tendência de Pontiero em recorrer a explicitações sintáticas ao utilizar a forma say.

Já a maior utilização da forma saying $(80,0 \%)$ com o conectivo zero parece mostrar-se divergente do padrão esperado. Nesta etapa do estudo, não é possivel, no entanto, fazer generalizações, havendo a necessidade de exploração em corpus mais extenso e variado contendo outras traduções do tradutor literário alvo de análise. 
Em contrapartida, as formas says $(83,3 \%)$, said $(68,8 \%)$ no TT analisado, ao registrarem porcentagens mais altas do conectivo zero, estão mais próximas do padrão de textos originalmente escritos em inglês, representados no BNC. Essa diferença no uso apresentada por Pontiero em relação a says e said, porém, parece estar em conformidade com as porcentagens mais baixas dessas duas formas, observadas nos TTs para o inglês representados no TEC, conforme a Tabela 1 , acima.

\section{Alguns padrões estilisticos preferenciais do tradutor em expressões fixas e semi-fixas}

Além das diferenças em termos de freqüência de thate zero, um exame mais detalhado dos dados sugere que também há diferenças em termos de preferências por padrões com discurso indireto em relação às formas do verbo de elocução SAY. Mesmo com um corpus de tamanho pequeno, alguns padrões de expressões fixas e semi-fixas mostraram proporções consideravelmente altas, como no caso de 15 ocorrências de that is to say num total geral de apenas 193.891 palavras do corpus de tradução.

A estrutura dominante, dada a sua freqüência mais elevada em Discovering the Word, é a da forma say e é a que mais apresenta padrões diferentes. Destaca-se, no Apêndice 2, amostra do uso de expressões fixas e semi-fixas envolvendo a forma say pela maior incidência, dentre elas: what I am trying to say, if I were to say, have nothing to say, I have to say, went on to say, needless to say, I can say, I would say, I cannot say. Ocorreu apenas uma construção empregando one como sujeito: one could say. Segundo Olohan e Baker (2000: 157), construções com one na função de sujeito seriam evitadas nas traduções e haveria maior utilização de construções passivas com said e o sujeito impessoal it (como em It is said that), por haver menos implicação em termos de registro. Por sua vez, aparecem outras construções correspondendo sintaticamente a formas de voz passiva, mas de construção mais simples: people say, they say. $\mathrm{O}$ uso de nomes próprios poderia ser resultante de uma estratégia de explicitação que favorece o uso de nomes específicos em oposição a pronomes pessoais, por serem estes menos específicos: Eva went on to say that we waste a great deal of energy. 
Quanto à forma says, nota-se uma incidência no uso do pronome reto na função de sujeito de says, como em: He says he has met me, ou em: Perhaps in response to something I may have written, she says that the writer, if genuine, always reveals herself. Seguem-se ocorrências em que o sujeito é um substantivo comum: My friend says she finds me amusing. No corpus de TT, não foi identificado nenhum caso de uso figurado de says com sujeito inanimado. Outrossim, não houve registro da forma says utilizada em expressões fixas e semi-fixas na tradução analisada.

Examinando a forma said, o maior número de ocorrências apresenta a construção said + zero como oração principal, colocada no início do período e iniciada por pronome sujeito, como em I said I would telephone her later. Há também incidências em que a oração subordinada com said é a segunda oração no período, e ligada à oração principal ou por meio de conjunções and, that: He approved of my questions and said he would expect me next morning, ou ligada por meio de pronomes relativos who, when, which: I was lying when I said orchids are obnoxious. Ao contrário do encontrado por Olohan e Baker (2000: 157), não se observou nenhum caso de voz passiva com discurso indireto. Como exemplo de expressões fixas e semi-fixas com said podem-se citar: nothing more to be said, I would have said, the things he said.

No tocante à forma saying, ocorrem expressões fixas e semifixas, como por exemplo: without saying a word, as I was saying. Também há incidência do vocábulo saying empregado como substantivo comum, no sentido de dito popular: as the saying goes.

A fim de examinar se a alta freqüência de expressões fixas e semi-fixas encontrada no TT não estaria sofrendo influência do respectivo TO, utilizei outro software, o MultiConcord. Por meio de alinhamentos obtidos com amostras de sentenças ou parágrafos contendo como nódulos as formas de SAY, foram feitas algumas observações iniciais, tendo sido identificadas uma incidência alta de acréscimos de expressões fixas e semi-fixas nesta tradução de Ponteiro.

Serve de exemplo, os dois fragmentos da crônica de 19 de agosto de 1967, respectivamente: "Tiresome children" $\leftarrow$ "As 
crianças chatas", abaixo, os quais foram extraídos e alinhados por meio da aplicação do programa MultiConcord em Discovering the World e A Descoberta do Mundo. Como se pode notar, Pontiero emprega duas expressões fixas: that is to say e that is, além de outras duas explicitações: like e something or someone, as quais não se encontram no TO de Clarice Lispector:

\footnotetext{
Discovering the World <- A Descoberta do Mundo 1967 -

P67 A cat did so much wailing during the night that I have rarely felt such compassion for the living. It sounded like grief, and in human and animal terms that is what it was. But could it have been sorrow, or was it 'searching', that is to say 'searching for'? For everything alive is searching for something or someone.

P67 Esta noite um gato chorou tanto que tive uma das mais profundas compaixões pelo que é vivo. Parecia dor, e, em nossos termos humanos e animais, era. Mas seria dor, ou era "ir", "ir para"? Pois o que é vivo vai para.
}

Levando em conta que os tradutores são escritores, então, tal qual os outros escritores, podem fazer uso especial de suas expressões preferidas. Contudo, em virtude do número significativo de expressões fixas e semi-fixas na tradução selecionada para compor o corpus principal, mostra-se possivel atribuir esse padrão de alta recorrência a uma característica particular e distintiva do estilo do tradutor em questão. Corrobora para isso o fato de um exame preliminar no respectivo TO de Clarice Lispector apontar serem potencialmente independentes do estilo da autora esses padrões de expressões fixas e semi-fixas empregados preferencialmente por Giovanni Pontiero.

Outra evidência que se poderia ainda levantar a respeito da hipótese de explicitação está no tamanho do texto. A contagem gerada pela ferramenta WordList Statistics mostra que a obra traduzida Discovering the World, com 193.891 palavras, é mais extensa do que A Descoberta do Mundo, com 171.687 palavras. Pelo princípio da explicitação, a maior extensão verificada no TT seria devido à maior quantidade de palavras empregadas pelo tradutor, para tornar, possivelmente, uma informação mais explícita, como, por exemplo, adições feitas por Pontiero iniciadas por marcadores explicativos: that is, that is to say, in other words.

Desta feita, ainda que o corpus desta investigação contenha apenas uma obra traduzida e a respectiva obra original, foi possivel a identificação de escolhas estilísticas do tradutor lite- 
rário Giovanni Pontiero, feitas consciente ou inconscientemente, as quais evidenciam freqüências altas no emprego do conectivo optional that com a forma say, bem como na utilização de expressões fixas e semi-fixas. Esses padrões estilísticos individuais, recorrentes e preferenciais, encontrados na tradução analisada, sugerem que o tradutor em questão vale-se freqüentemente de estratégias explicitadoras para conferir fluência ao texto de chegada. Outrossim, o alto índice de padrões de repetição de expressões fixas e semi-fixas sugere que o profissional selecionado para observação vale-se freqüentemente de estratégias simplificadoras para facilitar a compreensão da escritura clariciana para leitores de língua inglesa.

Dentre as limitações do presente estudo, o requisito de compilação de um corpus extenso é inerente à abordagem baseada no uso de corpora de TTs como uma ferramenta para investigar e descrever traduções e o comportamento de tradutores dentro dos estudos da tradução. Por essa razão, Olohan e Baker atestam que as "investigações levadas a feito até o momento podem ser descritas como exploratórias e em pequena escala" (Olohan \& Baker, 2000: 141). Além do problema do tamanho do corpus, existem variáveis dificeis de serem controladas, referentes à poética da narrativa brasileira, aos diversos recursos estilísticos da autora, normas de tradução, coerções do mercado editorial, escolha pelo tradutor do tipo de material a ser traduzido.

Por outro lado, este estudo procurou mostrar que uma investigação do estilo individual de um tradutor literário é, em princípio, exeqüivel e interessante. Dado que os teóricos desta área de estudo têm demonstrado objetivamente que a tradução é uma atividade que envolve criação e não apenas reprodução, tornam-se relevantes pesquisas a respeito do estilo do tradutor, ou pelo menos do tradutor literário, realizadas sob a ótica do tradutor em vez da verificação de o estilo do autor ter sido adequadamente ou não transposto na tradução. Em sendo uma atividade criadora e criativa, como de fato acreditamos, então em algumas partes ao longo do novo texto o tradutor deixa suas marcas individuais, distintivas e preferenciais. A dificuldade, contudo, reside no desenvolvimento de uma metodologia coe- 
rente para investigar tais marcas, e também distinguir o que é próprio de cada um dos dois 'autores', próprio dos dois textos, das duas línguas / culturas envolvidas.

\section{Referências bibliográficas}

\section{Material empregado no corpus}

LISPECTOR, C. $(1984,1987)$ A descoberta do mundo. Rio de Janeiro: Nova Fronteira, 781 p. Tradução de Giovanni Pontiero. Discovering the world. Manchester: Carcanel Press, 1992, 652 p. Disponivel no TEC fn000006.txt.

\section{Textos e obras gerais}

BAKER, M. (1993) Corpus Linguistics and Translation Studies: implications and applications. In: BAKER, M.; FRANCIS, G. \& TOGNINI-BONELLI, E. (orgs.). Text and technology: in honour of John Sinclair. Amsterdã e Filadélfia: John Benjamins, p. 233-250.

- (1995) Corpora in Translation Studies: an overview and some suggestions for future research. Target. 7:2, p. 223-243.

. (1996) Corpus-based translation studies: the challenges that lie ahead. In: SOMERS, H. (org.). Terminology, LSP and translation studies in language engineering, in honour of Juan C. Sager. Amsterdã e Filadélfia: John Benjamins, p. 175-186.

- (2000) Towards a methodology for investigating the style of a literary translator. Target. 12:2, p. 241-266.

. (2004) A corpus-based view $n$ of similarity and difference in translation. International Journal of Corpus Linguistics. 9:2), p. 167-193. BERBER SARDINHA, A. P. (2004) Lingüística de corpus. São Paulo: Manole.

BRITISH National Corpus: written corpus design specification (1991) OUP Promotional Document, 2 set.

BURNETT, S. (1999) A corpus-based study of translational English. Dissertação (Mestrado em tradução), CTIS/ UMIST, Manchester. 
EVEN-ZOHAR, I. (1978, 2000) The position of translated literature within the literary polysystem. In: HOLMES, J. S.; LAMBERT, J \& van den BROECK, R. (orgs.). Literature and translation. Leuven: ACCO, p. 117-127. [Versão revisada em VENUTI, L. (org.). The translation studies reader. Londres e Nova York: Routledge, p. 192-197].

HERMANS, T. (1996) The translator's voice in translated narrative. Target. 8:1, p. 23-48.

HOUSE, J. (1977, 1981, 1997) Translation quality assessment: a model revisitorg. Tubingen: Gunter Narr.

MULTICONCORD: the Lingua Multilingual Parallel Concordancer for Windows, Birmingham: University of Birmingham.

OLOHAN, M. \& M. BAKER (2000) Reporting that in translated English: evidence for subconscious processes of explicitation?. Target. 1(2), p. $141-158$.

SCOTT, M. (1998) WordSmith Tools. Software para Windows 3.1, 95 e 98. Oxford University Press. Versão 'demo', com funções limitadas, disponivel em: <http://www.liv.ac.uk/ ms2938>.

SINCLAIR, J. M. (1991) Corpus, concordance, collocation. Oxford: Oxford University Press.

TAGNIN, S. E. O. (2001) COMET: um COrpus Multilingüe para Ensino e Tradução. São Paulo: USP. Manuscrito.

TEC - Translational English Corpus. In: Centre for Translation and Intercultural Studies - CTIS. University of Manchester Institute of Science and Technology - UMIST. Disponivel em: <http:// ubatuba.ccl.umist.ac.uk/tec/>.

TOURY, G. (1978, 2000) The nature and role of norms in literary translation. In: HOLMES, J. S.; LAMBERT, J \& van den BROECK, R. (orgs.). Literature and translation. Leuven: ACCO, p. 83-100. [Versão revisada em VENUTI, L. (org.). The translation studies reader. Londres e Nova York: Routledge, p. 198-211].

TYMOCZKO, M. (1998) Computerized corpora and the future of Translation Studies. Meta. 43:4. p. 652-659.

VENUTI, L. (1992) Simpatico. Trabalhos em lingüistica aplicada. 19. jan-jun., p. 21-39.

. (1995) The translator's invisibility. Londres e Nova York: Routledge. . (1998) The scandals of translation. Londres e Nova York: Routledge.

WOOLLS, D. Multiconcord: the Lingua Multilingual Parallel Concordancer for Windows, Birmingham: University of Birmingham. 
WORDSMITH Tools. Software for Windows 3.1, 95 e 98. Oxford University Press. 'Demo' version, with limited functions, available at: <http:/ /www.liv.ac.uk/ ms2938>.

\title{
APÊNDICE 1 \\ CONCORDÂNCIAS COM ESTRUTURAS DO VERBO SAY GERADAS NA TRADUÇÃO FEITA POR PONTIERO DA OBRA Discovering the World, DE CLARICE LISPECTOR
}

\author{
P-L_DW- say WordSmith Tools -
}

$\mathrm{N} \quad$ File Concordance

1 discov 1 artment which was furnished. But let me say first of all that when reality is

2 discov 1 about searching for that truth. People say he died in the name of truth, but

discov 1 nsist, although it would not be true to say he was pleased. We confronted each

discov 1 how to prepare octopus, but I cannot say I like those tentacles. However, the

discov 1 sense I have ever written, and I dare say I have written a great de al in my ti

discov 1 rises me any more. I even hear people say I am aggressive whilst I have always

discov 1 oing to say, for heaven's sake? I shall say I picked a daisy and put it in the

discov 1 sappointed. It would have been a lie to say I was enjoying that bubble-gum. An

discov 1 . - All right, I believe you when you say I'm the first girl you've fallen in

discov 1 effective form of protest. 13 - I can say in all sincerity that I like every on

discov 1 KE I know it sounds rather fatuous to say in the middle of spring that I know

discov 1 to hold back her tears. Why did you say in your latest article that you are

discov 1 t. At the age of ten. - How would you say inspiration comes in your case? -

discov 1 le on my skin. My father always used to say it was good for one's health. In $\mathrm{f}$

discov 1 iration comes in your case? - I would say it comes in all sorts of ways. But I

discov 1 he leads the life of a poet.' One could say, judging from the few words I hear

discov 1 ous and self-controlled? One might also say that this word is contradictory ev

discov 1 of a Drunken Woman', I can confidently say that I have rarely enjoyed anythin existed. - Wha discov $\sim 1$ en her any choice, and then she could say that she had been forced to obey. At 
21 discov 1 from the few words I heard the couple say, that they both led, leaving aside a

22 discov 1 carnation? - I don't know. The Hindus say that only those who are conscious of

23 discov $\sim 1$ mines our perception of reality. When I say that Lucrécia Neves builds the cit

24 discov 1 intuition. And when, dear God, can one say that the heart is pure? For purity

25 discov 1 TITLED How could anyone have dared to say that I vegetate rather than live? Ju

26 discov 1 to know about Félicien. Suffice it to say that after dreaming about wine one $\mathrm{S}$

27 discov 1 e typical of Brasília. - If I were to say that Brasilia is pleasant, you would

28 discov $\sim 1$ ars a smile - no, no, I am not about to say that his smile lights up his entir

discov 1 things more clearly: was he trying to say that ... that I was a hidden treasur

30

discov 1 el purified. It simply is not true to say that people cannot be helped. The me

discov $\sim 1$ iendship and hospitality. Érico used to say that his happiest memories of Wash

discov 1 ? - no - it would be more accurate to say that the excited bee and flower were

discov 1 that I like the city. But if I were to say that Brasilia is the image of my i

34 discov 1 an impulse. It would be misleading to say that this impulse held the woman $b$

discov $\sim 1$ hose great moist eyes. Eva went on to say that we waste a great deal of energy

36

discov 1 a weak gesture with my hands as if to say there was nothing I could do, while

37

discov 1 The colour of stone, one could almost say they have no colour. Nova Friburgo

discov $\sim 1$ thing. When feeling nervous, the French say they are sur le qui-vive. We are $\mathrm{p}$

39 discov 1 unced with great excitement: - People say we're in the twentieth century, is $t$

40 discov $1 \mathrm{r}$ feet.' Is that what you mean when you say you are wary of savoir-faire? -

41 discov 1 a kiss. - Didn't I tell you? Didn't I say you're looking sad? You can't stop $\mathrm{k}$

P-L_DW- says

N File Concordance Set

1 discov $1 \mathrm{n}$ in saying I find you beautiful.' He says he has met me, but I have a hopeles

2 discov 1 In semi-darkness. LABYRINTH When he says he is wasting time, the others unde

3 discov $\sim \mathrm{m}$ and looks for the person his mother says he is calling; he turns his head an

4 discov 1 axed in each other's company. My friend says she finds me amusing. That does $\mathrm{n}$

5 discov 1 e month. She was most grateful. She now says she wants to find herself a boyfr

6 discov 1 to something I may have written, she says that the writer, if genuine, always 
P-L DW-said

$\mathrm{N}$ File Concordance

1 discov 1 omfort. He approved of my questions and said he would expect me next morning.

2 discov 1 lled The Passion According to G.H. He said he liked the title. I know he wou or-bell rang. I thought to myself: he said he would telephone

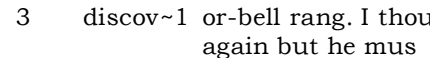

4 discov 1 ioned this idea to one of my boys who said he did not want to meet Chico. I as

5 discov 1 en was bound to have: a weak heart. I said I would telephone her later. What a

discov 1 phone again at six in the evening. He said it would be difficult. But he calle

discov 1 that day and I offered her some. She said it was delicious and asked for a se

8 discov 1 of its nutrition. I was lying when I said orchids are obnoxious: I adore them

9 discov 1 en I wrote an article about animals I said, quite intuitively, that the turtle

10 discov 1 have the proof? Not that she had ever said she did not go out on her own or me

11 discov 1 had given the book to Dr Lourival. She said she had and, on reading the dedic

12 discov 1 know if I considered it important. I said that I did, but not nearly as impor

13 discov 1 pted by an article of mine in which I said that I preferred to be unpleasant.

14 discov 1 at's all I ask when I die. Then the man said that the rain was gold, and no on rent. 14 September WRITING I once said that writing is a curse. I cannot $r$

16 discov 1 er. You wouldn't buy me one because you said they were dangerous, that the thi

P-L_DW-saying

N File Concordance Set

1 discov 1 ngs he had written. I shocked them by saying I could never bear to re-read any

2 discov 1 nd downs, but I have no hesitation in saying I find you beautiful.' He says

3 discov 1 - Fear. What a pity I cannot reply by saying it is hope. - What is the best

4 discov 1 process is difficult? But that is like saying that the extremely capricious a

5 discov $\sim 1$ ndless scope. It can be anything from saying the wrong thing at the worst poss 


\section{APÊNDICE 2}

AMOSTRA DE EXPRESSÕES FIXAS E SEMI-FIXAS COM FORMAS DO VERBO SAY GERADAS NA TRADUÇÃO FEITA POR PONTIERO DA OBRA Discovering the World, DE CLARICE LISPECTOR

P-L_ DW-cluster: fixed \& semi-fixed expressions with SAY

\begin{tabular}{|c|c|}
\hline $\begin{array}{l}\mathrm{N} \\
2\end{array}$ & $\begin{array}{l}\text { cluster Freq. } 6 \\
\text { what i am trying to say }\end{array}$ \\
\hline $\mathrm{N}$ & cluster Freq. 5 \\
\hline 4 & if $\mathrm{i}$ were to say \\
\hline 13 & i can say no more \\
\hline 14 & i shall say no more \\
\hline $\mathrm{N}$ & cluster Freq. 4 \\
\hline 1 & that is to say \\
\hline 2 & have nothing to say \\
\hline 4 & i have to say \\
\hline 10 & went on to say \\
\hline 11 & how does one say \\
\hline 16 & nothing more to say \\
\hline 13 & all i can say \\
\hline 14 & all i could say \\
\hline 23 & how does one say \\
\hline 27 & i really cannot say \\
\hline $\mathrm{N}$ & cluster Freq. 3 \\
\hline 6 & needless to say \\
\hline 7 & i can say \\
\hline 12 & i would say \\
\hline 13 & i cannot say \\
\hline 14 & one could say \\
\hline 20 & say no more \\
\hline 24 & used to say \\
\hline 30 & let us say \\
\hline 44 & more to say \\
\hline 45 & difficult to say \\
\hline 48 & prepared to say \\
\hline 49 & really cannot say \\
\hline 74 & true to say \\
\hline $\mathrm{N}$ & cluster Freq. 2 \\
\hline 48 & one say \\
\hline 30 & people say \\
\hline 36 & let's say \\
\hline 196 & they say \\
\hline
\end{tabular}

P-L_DW-cluster: fixed \& semi-fixed expressions with SAYS

Raw frequency subtotal: 
P-L_ $\boldsymbol{D} \boldsymbol{W}$-cluster: fixed \& semi-fixed expressions with SAID

$\begin{array}{lll}\text { N } & \text { cluster Freq. } 5 & \\ 1 & \text { nothing more to be said } & 4 \\ \text { N } & \text { cluster Freq. } 4 & 3 \\ 2 & \text { as i said before } & 2 \\ 9 & \text { i would have said } & 2 \\ 11 & \text { the things he said }\end{array}$

P-L_DW -cluster: fixed \& semi-fixed expressions with SAYING

$\mathrm{N} \quad$ cluster Freq. 4

2 without saying a word 3

3 as i was saying 2

4 as the saying* goes $3 *$ (Noun) 